\title{
The influence of automatic associations on preference for milk type
}

\author{
W. S. Harwood ${ }^{1}$ and M. A. Drake ${ }^{1 *}($ (]) \\ ${ }^{1}$ Department of Food, Bioprocessing, and Nutrition Sciences, Southeast Dairy Foods Research Center, North Carolina State University, \\ Raleigh, 27695
}

\section{ABSTRACT}

In recent decades, organic milk has been an exception to the trend of decreased fluid milk consumption in the United States. However, the reasons behind consumer preference for organic milk over conventional and other milk types are ill-defined. The objective of this study was to use an implicit association test (IAT) and primed and unprimed preference testing to determine if fluid milk consumer preferences for milk types are influenced by implicit biases and, if so, to define these biases within the context of the consumer sensory experience. Self-reported fluid milk consumers $(n=473)$ participated in online IAT exercises where pairwise comparisons of milk types (conventional, organic, local, pasture-raised) were measured on both positive and negative dimensions related to cow-welfare, sustainability, health, trust, safety, and quality. Latency times from IAT responses were subsequently transformed into standardized D-scores to categorize bias effect sizes. Additionally, fluid milk consumers $(\mathrm{n}=174)$ participated in preference tests that compared commercial milks representing different milk types through presentations where milk type was shown (primed) or hidden (unprimed). Following preference tests, consumers were asked to explain their preferred sample using check-allthat-apply tasks. Analysis of IAT results showed that conventional milk was more associated with negative dimension descriptors compared with organic, local, and pasture-raised milks. Additionally, a positive bias in favor of organic milk was expressed when compared with pasture-raised milk offerings; however, no differences were found in pairwise comparisons of other nonconventional milk type pairings, suggesting that consumers may conflate these designations. Blinded preference testing showed that milk preferences were largely dictated by flavor, with consumers differentiating milk types based upon flavors related to packaging, pasteurization method, and feeding systems. In primed evaluations, consumers generally expressed preferences

Received June 7, 2020.

Accepted July 22, 2020.

*Corresponding author: mdrake@ncsu.edu that aligned with their explicit beliefs, and flavor considerations appeared to be a secondary differentiator of preference. Based on these results, conventional milk is associated with negative implicit beliefs related to production and product quality more often than other milk types, which was reflected in IAT evaluations and primed preference tests. However, the blinded tastings suggested that conventional milk was preferred, or competitive with, other milk types based on flavor alone. Findings from this study suggest consumer differentiation and preference of milks is significantly affected by perceptions and beliefs related to milk type. In particular, conventional milk was associated with relatively few unique belief descriptions, indicating strict utilitarian consumer categorization. Organic and local milks were comparatively associated with greater care for nutrition, sustainability, animal welfare, and local farm support. These results demonstrate a need for greater education related to conventional milk offerings to dispel factors influencing negative implicit bias. Furthermore, improving product narrative via label information and alignment with locality and regionality were identified as possible opportunities for improving consumer sentiments related to conventional milk. A more thorough understanding of these attributes may reinforce stated beliefs more effectively and stave off consumer losses to plant-based alternatives that fulfill similar beliefs.

Key words: milk, organic, implicit, bias, local, consumer

\section{INTRODUCTION}

Consumption of fluid milk has steadily declined the last several decades, coinciding with increased consumption of plant-based alternatives (Haas et al., 2019; USDA ERS, 2019). While this trend is problematic for milk producers, fluid milk is still widely considered a staple food item in many American households (McCarthy et al., 2017). Furthermore, commercial fluid milk offerings have grown considerably over the last few decades to meet consumer needs. For example, growing consumer concern about lactose intolerance has been widely addressed through the production of lactose-free 
milks via lactase enzyme or ultrafiltration in such a way that multiple consumer sentiments may be addressed through available commercial milk options (Rizzo et al., 2020). Similarly, technological advances in packaging and increased thermal treatment have helped assuage consumer concerns related to shelf life of fluid milk, although application of higher heat treatments alter the sensory profile of milk (Gandy et al., 2008; Lee et al., 2017).

Although flavor preference is certainly a driver of ultimate acceptance, previous studies on milk and milk-alternative consumers have indicated that the primary reasons for choosing plant alternatives include greater perceived animal welfare, greater environmental sustainability, and better nutrition (McCarthy et al., 2017; Haas et al., 2019). However, nonconventional fluid milk offerings such those designated as organic, locally farmed, or pasture-raised may similarly comply to these consumer belief systems. In a survey of fluid milk consumers, those who exclusively consumed organic milk reported doing so largely because they perceived greater health and quality compared with conventional milk, and believed organic milk production was associated with greater support for local farms and farmers, higher animal welfare, and greater sustainability (Harwood and Drake, 2018). Furthermore, findings from Harwood and Drake (2020) reported that some milk consumers valued the increased shelf life of organic and pasture-raised milks that were ultrapasteurized, though consumers were largely unaware these qualities were associated with an increased thermal processing treatment. Cardoso et al. (2016) additionally reported that pasture access, adherence to organic practices, and engagement with local farming were cited as essential parts of an "ideal" dairy farm in open-ended consumer surveys, reinforcing consumer attraction to such features. Quantification of these sentiments as they relate to commercial fluid milks has been extensively studied; however, these findings are often limited to willingness-to-pay and price-modeling studies (Wolf et al., 2011; Kühl et al., 2017). As such, a more detailed understanding of consumer perceptions related to different dairy systems is warranted to inform product marketing, education initiatives, and potential benefits and risks of transitioning farming practices.

Several methods exist for capturing consumer beliefs, preferences, and motivations; however, this plethora of tools can generally be condensed to either implicit or explicit measures. Explicit beliefs generally refer to conscious attitude or beliefs that are directly expressed by an individual upon introspection, whereas implicit beliefs are characterized as subconscious, underlying, unknown, or otherwise unable to be readily expressed (Friese et al., 2006). Explicit belief measures commonly used for food products include direct rating (hedonic, magnitude estimation, Likert, semantic differential, or agreement scales), choice procedures (preference tests), or free-response to capture conscious motivations and preferences (Moskowitz and Sidel, 1971; Lim, 2011). Although explicit measures are incredibly useful in modeling and defining preferences, consumers may hold subconscious implicit motivations that also guide their ultimate behavior (Friese et al., 2006). Implicit measures can be collected in a variety of ways, including physical responses or multinomial choice experiments (Ares et al., 2013; Harwood and Drake, 2019). Furthermore, the joint application of explicit and implicit measurements may provide a more holistic understanding of consumer preferences and may help correct for incorrect assumptions or misleading conclusions. The present study proposes the application of this dual approach to understand consumer preferences as they relate to fluid milk farming systems from an explicit and implicit perspective, coupled with insights that address both sensory and conceptual motivations.

\section{MATERIALS AND METHODS}

\section{Experimental Overview}

Implicit beliefs, sensory preferences, and primed preferences associated with fluid milk types (conventional, organic, locally farmed, pasture-raised) were assessed using a 2-part study. In the first part of the study, an online implicit association test (IAT) was used to compare fluid milk type pairings to evaluate the presence or absence of implicit biases. The IAT participants $(\mathrm{n}=473$ total $)$ were asked to complete 3 of a possible 6 IAT tests (representing the 6 possible milk type pairings). Participants who completed all 3 assigned IAT exercises were entered into a drawing to receive a gift certificate to a local store. In the second part of the study, fluid milk consumers ( $\mathrm{n}=174$ total) were recruited to participate in a series of 6 preference tests, representing the 6 possible milk type pairings. Preference-test participants were randomly assigned to either a blinded or primed (milk types disclosed) group to understand the effect of milk type on preferences. Preference-test participants were compensated with a $\$ 10$ gift certificate to a local store. All procedures were conducted in compliance with North Carolina State University Institutional Review Board regulations.

\section{Implicit Association Test}

Six online IAT surveys, each representing a possible milk type pairing, were constructed using the iatgen web-based tool developed by Carpenter et al. (2019) 
Table 1. Example overview of implicit association test structure $^{1}$ (conventional vs. organic milk example)

\begin{tabular}{lllll}
\hline Block & No. of trials & Category & Left-key response $^{2}$ & Right-key response $^{2}$ \\
\hline 1 & 20 practice & Target sorting & Conventional milk images & Organic milk images \\
2 & 20 practice & Category sorting & Positive & Negative \\
3 & 20 practice & Combined block & Conventional milk images + positive & Organic milk images + negative \\
4 & 40 collected & Combined block & Conventional milk images + positive & Organic milk images + negative \\
5 & 40 practice & Category sorting & Organic milk images & Conventional milk images \\
6 & 20 practice & Combined block & Organic milk images + positive & Conventional milk images + negative \\
7 & 40 collected & Combined block & Organic milk images + positive & Conventional milk images + negative \\
\hline
\end{tabular}

${ }^{1}$ Targets were 6 photographs of commercial products representing each tested milk type (conventional, organic, locally farmed, pasture raised). Categories were (1) positive = happy cows, sustainable, healthy, trustworthy, safe, high quality and (2) negative = unhappy cows, wasteful, unhealthy, misleading, unsafe, low quality.

${ }^{2}$ Presentation of targets and categories was balanced for position among participants (Nosek et al., 2005).

${ }^{3}$ Lengthened block 5 practice set is intended to clear learned behavior based on positioning of earlier blocks (Nosek et al., 2005).

and were hosted on the Qualtrics (Provo, UT) software platform. Each IAT survey consisted of 2 milk-type targets, which were each represented by 6 images of commercial fluid milks corresponding to the given type, and 2 categories, representing positive and negative attributes related to milk production. Positive category attributes were the following: happy cows, sustainable, healthy, trustworthy, safe, high-quality. Negative category attributes were the following: unhappy cows, wasteful, unhealthy, misleading, unsafe, low-quality. Selection of category attributes was intended to capture multiple angles related to milk production (e.g., animal welfare, sustainability, health, quality), and terminology was influenced by previous studies related to fluid milk and milk production beliefs (Cardoso et al., 2016). An additional survey was composed to capture basic demographic and usage questions before participation in the IAT surveys, as well as to screen out unqualified participants. Participants who were at least $18 \mathrm{yr}$ of age and reported consumption of fluid milk at least a few times per month were considered for participation in the IAT tests. Following completion of demographic and usage questions, qualified participants were randomly assigned 3 of a possible 6 links, with each link corresponding to a different IAT.

Each IAT consisted of 7 blocks of sorting tasks, as described by Carpenter et al. (2019), which were randomized and balanced to account for order effects (Nosek et al., 2005). An example overview for the conventional versus organic milk type pairing IAT can be found in Table 1. For each test, participants were introduced to the stimuli (milk type images and positive and negative text attributes) before beginning the IAT exercise. For each block, respondents were asked to sort (using the "e" key to sort left, or the "i" key to sort right) stimuli to the left or right side of their screen to match the appropriate category, which was fixed to a given side. For example, the screen may have shown an image of an organic milk in the middle of the screen, with the term "Organic" appearing on the left side of the screen, and "Conventional" appearing on the right side of the screen. The panelist would then need to press the "e" key to sort the image correctly to the "Organic" category. In the case of an incorrect answer, an error message immediately appeared, and panelists were only able to move on once the stimulus was correctly answered. Within the fourth and seventh blocks (scored combined blocks), latency time for each sorted stimulus was recorded. In the event of an incorrect answer, latency time was recorded once the stimulus was correctly sorted, with no penalty (Greenwald et al., 2003). For the purposes of replicating these methods, or adopting the approach for IAT survey design described in the present study, the reader is directed to review Carpenter et al. (2019) - a comprehensive overview on survey-based IAT design using the iatgen web tool.

\section{Preference Testing}

Self-reported fluid milk consumers $(\mathrm{n}=174$ total) were recruited to participate in 2-alternative forcedchoice preference tests on commercial milks representing different fluid milk types. Milks chosen for the study represented conventional (Harris Teeter $2 \%$; store brand, HTST pasteurization, high-density polyethylene jug), organic (Harris Teeter Organic 2\%; store brand, ultrapasteurized, paperboard carton), locally farmed (Maple View Farms 2\%; HTST pasteurization, glass bottle), and pasture-raised (Organic Valley Grassmilk $2 \%$; national brand, ultrapasteurized, paperboard carton) milk types. Upon arrival, panelists were randomly assigned to either a blinded (unprimed) cohort $(\mathrm{n}=87)$, or a primed cohort $(n=87)$. Panelists then proceeded to perform 6 two-alternative forced-choice preference tests on each possible milk type pairing. Presentation of milk pairings was randomized and balanced for sample position across consumers, and evaluations were performed and collected on an iPad interface hosting Compusense 
Cloud software (Guelph, Ontario). Prior to serving, each container of commercial milk used in the study was screened by 2 highly experienced sensory experts (each with $>500 \mathrm{~h}$ of experience with trained panel profiling and judging dairy defects on milk and dairy products) to ensure that there were no atypical flavors or quality defects (e.g., due to light oxidation or spoilage) present. Milks were served at approximately $4^{\circ} \mathrm{C}$ in 177-mL white Styrofoam (Dow Chemical Company, Midland, MI) cups labeled with 3-digit blinding codes. For blinded panelists, only the 3-digit blinding codes were displayed. Primed panelists similarly received samples with 3-digit blinding codes, but were also made aware of what the corresponding milk type was for each sample using text labels on the data collection interface. Text descriptions of milk type for primed consumers disclosed the milk type designation (conventional, organic, locally farmed, pasture-raised), but made no mention of brands, logos, or other label features. Once sample pairs were received, panelists were instructed to taste both samples, left to right, before deciding which sample they preferred. Following evaluation of each milk pair, a 3-min rest time was enforced, during which time panelists were instructed to cleanse their palate with water and an unsalted cracker. Once finished with evaluation of all 6 milk preference tests, panelists were asked to identify what features typically influenced their purchase of fluid milk using a list of 22 features in a check-all-that-apply (CATA) format.

\section{Data Analysis}

Following data collection, raw latency times from sorting tasks in the IAT survey were subjected to cleaning and analysis using the iatgen web-based analysis tool (Carpenter et al., 2019). Latency times over 10,000 ms were scored as missing, as were observations from participants who had $>10 \%$ trials under $300 \mathrm{~ms}$ (indicating a "click-thru" series of responses). Cleaned data were used to determine D-scores for each respondent, using the algorithm described by Greenwald et al. (2003). When combined with the positive descriptors category, lesser latency times (shorter response times) for targets (milk types) compared with when those same targets were combined with the negative descriptors category, were indicative of positive implicit biases. Additionally, the same was true for the other milk type concurrently assessed, allowing for a relative measure of implicit bias for each milk type pairing.

To calculate D-scores, within-person difference scores were first calculated by determining the mean latency time of combined-block practice results (blocks 3 and 6 ) and combined-block scored results (blocks 4 and 7). Next, these mean values were each divided by their as- sociated standard deviations, and the 2 resulting values were averaged to create a D-score, similar in nature to calculation of Cohen's $d$ for determining effect size. Individual D-scores were used to determine population statistics for each IAT (mean, SD, Cohen's $d$ ), and were also stratified based on magnitude to describe general strength of observed biases as nonexistent, slight, moderate, or strong (Greenwald et al., 2003; Rooth, 2010). We conducted ANOVA of D-scores with sex, age, presence of children, annual household income, ethnicity, and stated milk type preferences as independent variables that were subsequently used to investigate demographic determinants of milk type bias. Milk type preferences were asked in a CATA format, with conventional, organic, locally farmed, and pasture-raised milk types shown as available options. Results from this CATA question were subsequently condensed to define consumers as exclusive conventional milk consumers (only conventional chosen), exclusive nonconventional consumers (only nonconventional options chosen), or mixed option consumers (both conventional and nonconventional consumption habits).

Blinded and primed consumer preference-test results were tabulated and subsequently analyzed using the binomial test $(\alpha=0.05)$ to determine if significant differences in preference existed between milk types. Additionally, relationships between milk type preferences (both blinded and primed) and factors influencing fluid milk purchase (CATA) were assessed using the multiple correspondence analysis $(\mathbf{M C A}$; rows $=87$ panelists for each test condition, columns $=6$ preference-test results; 22 CATA features related to belief systems and purchase preference were included as supplemental variable columns) module within XLSTAT version 2020.1.2 (Addinsoft, Paris, France). Coordinates derived from MCA were subjected to agglomerative hierarchical clustering using a dissimilarity matrix with Euclidean distance, Ward's agglomeration method, and entropybased truncation of segments to effectively communicate connections between preference-test results and CATA-stated beliefs.

\section{RESULTS AND DISCUSSION}

\section{Implicit Association Test}

Across the 6 IAT tests, approximately $8.4 \%$ of evaluations were dropped due to incompletion or excessive speed within exercises (Table 2). Within the final data set, D-scores were calculated to assess whether an implicit bias existed between milk type pairings. It should be noted that a positive or negative $\mathrm{D}$-score is indicative of a directional implicit bias in favor of the milk type associated with linked direction. For example, within 
the conventional and organic milk type pairing, conventional was designated as the positive $(+)$ direction milk type and organic was designated as the negative $(-)$ direction milk type. In this case, the negative D-score for this pairing indicated an implicit bias directionally in favor of the organic milk type designation. An overview of positive and negative direction designations within each milk type pairing can be found in Table 2.

Analysis of IAT D-scores showed conventional milk was consistently associated with negative milk production attributes and farm practices, compared with organic ( $\mathrm{D}=-0.26, P<0.001, d=-0.46)$, locally farmed $(\mathrm{D}=-0.28, P<0.001, d=-0.48)$, and pasture-raised $(\mathrm{D}=-0.23, P<0.001, d=-0.38)$ milk types (Table 2). Evaluation of effect sizes and stratified individual results indicated that these biases may be interpreted as being moderate in nature, although strong biases in favor of nonconventional offerings were observed as the largest classification group for each test. Investigation of ANOVA main effects for each milk type pairing (results not shown) indicated that stated milk type preferences were a significant determinant $(P<0.05)$ of biases in conventional versus nonconventional milk type pairings, with respondents who reported strict conventional milk purchase habits expressing a lower $(P<0.05)$ bias in favor of organic, locally farmed, or pasture-raised milks, compared with respondents who reported strict nonconventional or mixed (conventional and nonconventional) milk type purchase habits. However, it should be noted that in each conventional pairing, respondents with strict conventional milk purchase habits directionally expressed biases against conventional milk. These results are consistent with findings from Hill and Lynchehaun (2002), who suggested that purchase habits of conventional milk consumers were more habitual compared with organic milk consumers who tend to exhibit more thoughtful and loyal purchase habits. Additionally, sex was reported as a significant determinant $(P<0.05)$ of bias for the conventional and pasture-raised pairing, with female respondents reporting a greater $(P<0.05)$ pro-pasture-raised bias compared with male respondents. This result can likely be attributed to the suggestion that pasture-based systems offer greater animal welfare, which has been shown to be a significantly more impactful issue for women than men (Vanhonacker et al., 2007).

Comparisons of nonconventional milk types yielded mixed results. A significant implicit bias, albeit "slight" in magnitude, was observed in favor of organic milk when compared with pasture-raised milk $(\mathrm{D}=0.10$, $P=0.001, d=0.22)$. However, no bias was found in organic and locally farmed $(\mathrm{D}=-0.02, P=0.541, d$ $=-0.04)$ or pasture-raised and locally farmed $(\mathrm{D}=$ $-0.03, P=0.409, d=-0.06)$ pairings. Analysis of
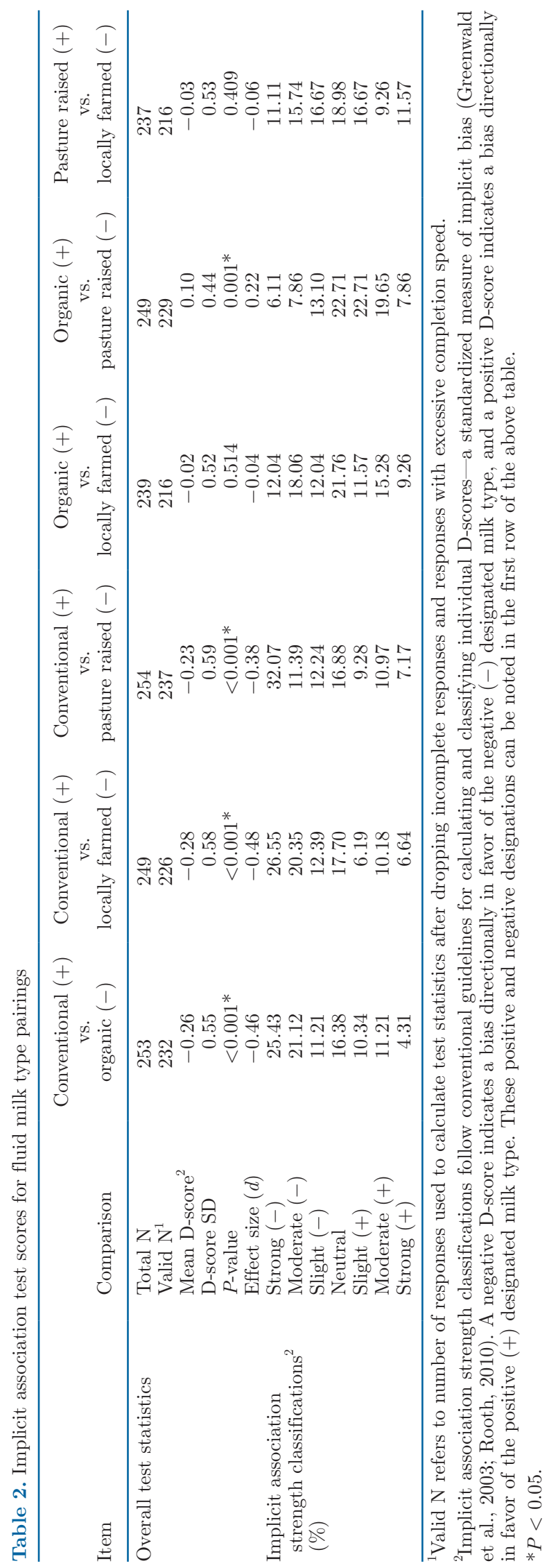
Table 3. Summary of milk type preference-test results from fluid milk consumers $(\mathrm{n}=174$ total)

\begin{tabular}{llclcc}
\hline Comparison & Treatment & Panelists & $\begin{array}{l}\text { 2-Alternative forced-choice } \\
\text { preference test results }\end{array}$ & $\begin{array}{c}P \text {-value } \\
(\text { binomial })\end{array}$ & $\begin{array}{c}P \text {-value } \\
\left(\chi^{2}\right)\end{array}$ \\
\hline Conventional vs. organic & Blind & 87 & Conventional-31, Organic-56 & $0.010^{*}$ & 0.436 \\
& Primed & 87 & Conventional-36, Organic-51 & 0.133 \\
Conventional vs. locally farmed & Blind & 87 & Conventional-45, Locally famed-42 & 0.830 & 0.094 \\
& Primed & 87 & Conventional-34, Locally farmed-53 & 0.053 \\
Conventional vs. pasture raised & Blind & 87 & Conventional-51, Pasture raised-36 & 0.133 \\
& Primed & 87 & Conventional-42, Pasture raised-45 & 0.171 \\
Organic vs. locally farmed & Blind & 87 & Organic-49, Locally farmed-38 & 0.830 \\
Organic vs. pasture raised & Primed & 87 & Organic-39, Locally farmed-48 & 0.284 & 0.129 \\
& Blind & 87 & Organic-50, Pasture raised-37 & 0.198 & 0.878 \\
Pasture-raised vs. locally farmed & Primed & 87 & Organic-51, Pasture raised-36 & 0.133 \\
& Blind & 87 & Pasture Raised-26, Locally farmed-61 & $<0.001^{*}$ & 0.113 \\
& Primed & 87 & Pasture Raised-36, Locally farmed-51 & 0.133 \\
\hline
\end{tabular}

$* P<0.05$.

main effects from the ANOVA model for the organic and pasture-raised pairing indicated the presence of children in the household and respondent ethnicity were significant determinants of bias. Specifically, a greater $(P<0.05)$ pro-organic bias was expressed for respondents who had children (compared with those who did not have children in their household), as well as for non-Caucasian respondents (compared with Caucasian respondents), consistent with trends reported in previous studies (Harwood and Drake, 2018). For the pasture-raised and locally farmed pairing, income and ethnicity were identified as significant determinants $(P$ $<0.05)$ of bias. Specifically, greater $(P<0.05)$ prolocally farmed bias was expressed by non-Caucasian respondents, as well as high-earners $(>\$ 80,000 / \mathrm{yr})$ compared with moderate-earners $(\$ 40,000-\$ 80,000$ / yr $)$, although there was no difference in bias $(P>0.05)$ compared with low-earners $(<\$ 40,000 / y r)$. Finally, for the organic and locally farmed pairing, no differences were noted among demographic groups, suggesting widespread belief that the 2 designations are interchangeable or share core features (Haas et al., 2013).

\section{Preference Testing}

Evaluation of preference-test results from blinded and primed consumer cohorts demonstrated sensory preferences, as well as the effects of milk type labeling for commercial fluid milks (Table 3). Within blinded evaluations, organic milk was preferred $(P<0.05)$ to conventional milk, and locally farmed milk was preferred $(P<0.05)$ to pasture-raised milk. Within primed evaluations, no significant preferences $(P>0.05)$ were noted for any milk type pairing. Furthermore, chisquared tests on proportions from blinded and primed tests revealed no significant differences in preference due to priming. Differences in preference observed from blinded preference tests suggest that meaningful differ- ences in sensory profiles exist in milks from different dairy systems; however, primed evaluations show that independent conclusions about these differences may be altered by the presence of milk type labeling.

\section{DISCUSSION}

When viewed independently, differences between blinded and primed preference-test evaluations for each milk type provided relevant, but limited, conclusions on the conceptual effect of milk type labeling. However, distinct differences in conceptual patterns were observed for each treatment type when aggregated in the context of stated beliefs and motivations (Figures 1 and 2 ). For the blinded cohort (Figure 1), differentiation of preference-test results and stated motivations behind fluid milk purchase were primarily defined along factor 1, which accounted for $54.71 \%$ of explained variability. In terms of milk type preference, differentiation along factor 1 indicated clear sensory differences between conventional and locally farmed milk, which loaded negatively, and organic and pasture-raised milk, which loaded positively. Sensory differences between conventional and pasture-based milk production systems are well-defined in previous literature, with pasture-based systems defined by higher sweet aromatic flavor, as well as green or grassy and mothball flavors; however, these sensory differences are often unnoticed by consumers (Croissant et al., 2007; Villeneuve et al., 2013). Though feed flavors certainly may have played an additive role in milk type differentiation by blind consumers, the conventional and locally farmed, and organic and pasture-raised groupings also differed in thermal processing and packaging materials, which have been cited as significant influences on consumer acceptance (Simon and Hansen, 2001; Lee et al., 2017).

When viewed jointly with self-reported influences and beliefs (CATA), analysis of clustered MCA coordinates 
from unprimed consumers reinforced these sensorybased groupings and showed that blinded preference patterns in favor of conventional or locally farmed milk (cluster 1) were clustered with relatively lower care for price, discounts and sales, brand, product narrative, or indications of farming practices (locally farmed, familyfarmed, validus-certified). However, those who exhibited such patterns were more likely to indicate fat content and package material were important purchase influencers (Figure 1). Alternatively, preference patterns in favor of organic and pasture-raised fluid milks (cluster 2) were clustered with relatively high care for brand, price, nutritional information, package size, shelf life, value-added features (rBST-free, DHA-fortified), product narrative, and farming practice attributes (locally farmed, family-farmed, validus-certified). As expected, blinded preference-test evaluations showed inconsistent trends in consumer belief patterns; however, select patterns suggested that subconscious associations between sensory profiles and product attributes may exist. For example, the higher cooked flavor (ultrapasteurized) and similar packaging of organic and pasture-raised milk (cardboard carton) may have proven familiar to organic milk consumers and helped them to align preference with their self-reported motivations, which often include suggestions of ethical consciousness (product narrative, locally farmed, family-farmed, validuscertified) and better perceived nutrition (Harwood and Drake, 2018; Scozzafava et al., 2020).

Results from primed cohort milk consumers (Figure 2 ) showed that milk type preference patterns were primarily differentiated along factor 1, which accounted for $47.62 \%$ of explained variability. Differentiation along factor 1 was defined by separation of locally farmed and organic (negative loading), pasture-raised (mixed loadings), and conventional (positive loading) milk type groupings, indicating a substantial deviation from the sensory-centric differentiation demonstrated by blind cohort consumers. These results suggest that in the case of fluid milk, attitudinal loyalty generally supersedes sensory preferences, a phenomenon noted in other food and beverage studies (Napolitano et al., 2013; Bernard and Liu, 2017). This is further evidenced through alignment of preferences to match self-reported beliefs. Inspection of clustered MCA coordinates revealed that these findings could be defined by 3 main response clusters.

For primed consumers, preference patterns in favor of locally farmed and organic (cluster 1) were associated with relatively higher care for discounts and sales, fat

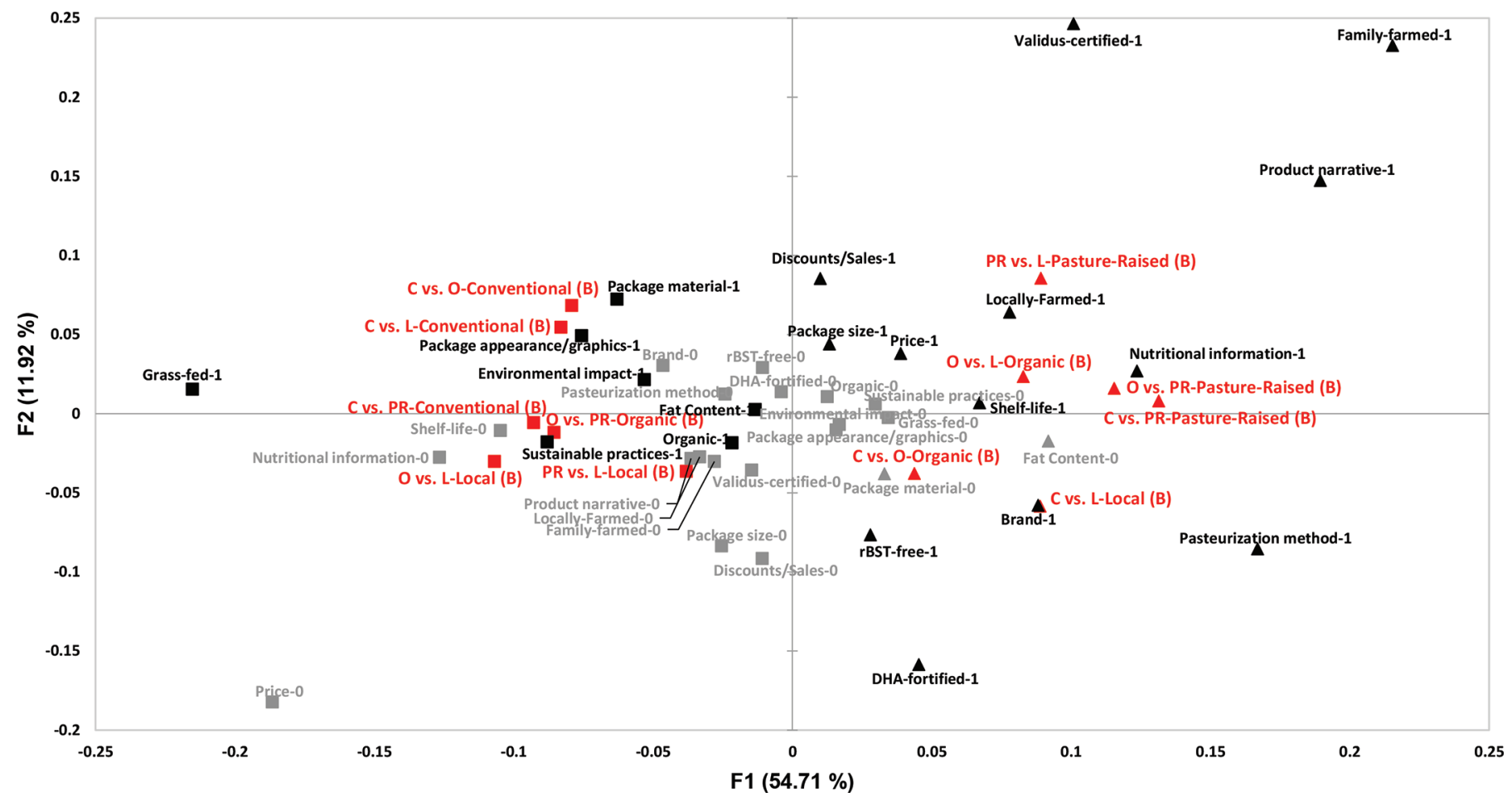

Figure 1. Multiple correspondence analysis of blinded cohort $(\mathrm{n}=87)$ preference-test results and self-reported influences on fluid milk purchase. $\mathrm{C}=$ conventional; $\mathrm{O}=$ organic; $\mathrm{L}=$ locally farmed; $\mathrm{PR}=$ pasture raised; $\mathrm{B}=$ blinded. Preference-test results are listed in the following order: pairing type, preference choice, priming treatment. $1=$ attribute was chosen in check-all-that-apply $(\mathrm{CATA})$ exercise, $0=$ attribute was not chosen in CATA exercise. $\mathbf{\square}=$ cluster 1 preferences and purchase influences; $\boldsymbol{\Delta}=$ cluster 2 preferences and purchase influences. 
content, nutritional information, value-added features (rBST-free, DHA-fortified), and farming practice attributes (locally farmed, family-farmed, validus-certified, sustainable practices). These results mirror previous studies that have identified benevolent belief systems and perception of greater product quality as being primary drivers for organic milk purchase (Harwood and Drake, 2018). Furthermore, these results corroborate IAT results of the organic and locally farmed milk type pairing, indicating that there is overlap in consumer perception regarding locally farmed and organic milk production systems (Haas et al., 2013). Unlike the first cluster, cluster 2 was defined by preference patterns against locally farmed milk, and coincided with higher regard for organic designation, pasteurization method, and product narrative. As these results directionally trended toward organic milk, but away from locally farmed milk, they likely indicated that organic milk consumers uniquely find relative added value in branding or narrative and extended shelf life-features not widely available (extended shelf life) or satisfied (branding tends to be minimalistic for local milk offerings) by locally farmed products. Previous research on fluid milk consumers has generally concluded that nonconventional fluid milk consumers are more inclined to consider product information and narrative than conventional milk consumers (Getter et al., 2015; Harwood and Drake, 2020). Furthermore, nonconventional consumer subgroups are differentiated by preferences for distinct product attributes (Harwood and Drake, 2020). As such, it is unsurprising that differences exist between organic and locally farmed milk preferences in terms of underlying belief systems and feature importance. Nevertheless, the relative similarity in consumer perception of organic and locally farmed milk, as evidenced in IAT and primed consumer evaluations, indicated that these milk types are largely conceptualized in the same fashion by the majority of consumers.

Finally, cluster 3 from primed consumers was generally defined by preference patterns in favor of conventional milk and was associated with higher care for price, brand, pasture-raised and grass-fed milk, package appearance and graphics, package material, and shelf life. In general, features captured by this cluster closely match sentiments associated with utilitarian milk consumer groups who express a higher purchase likelihood for conventional milk products and high price sensitivity (Rödiger and Hamm, 2015; Harwood

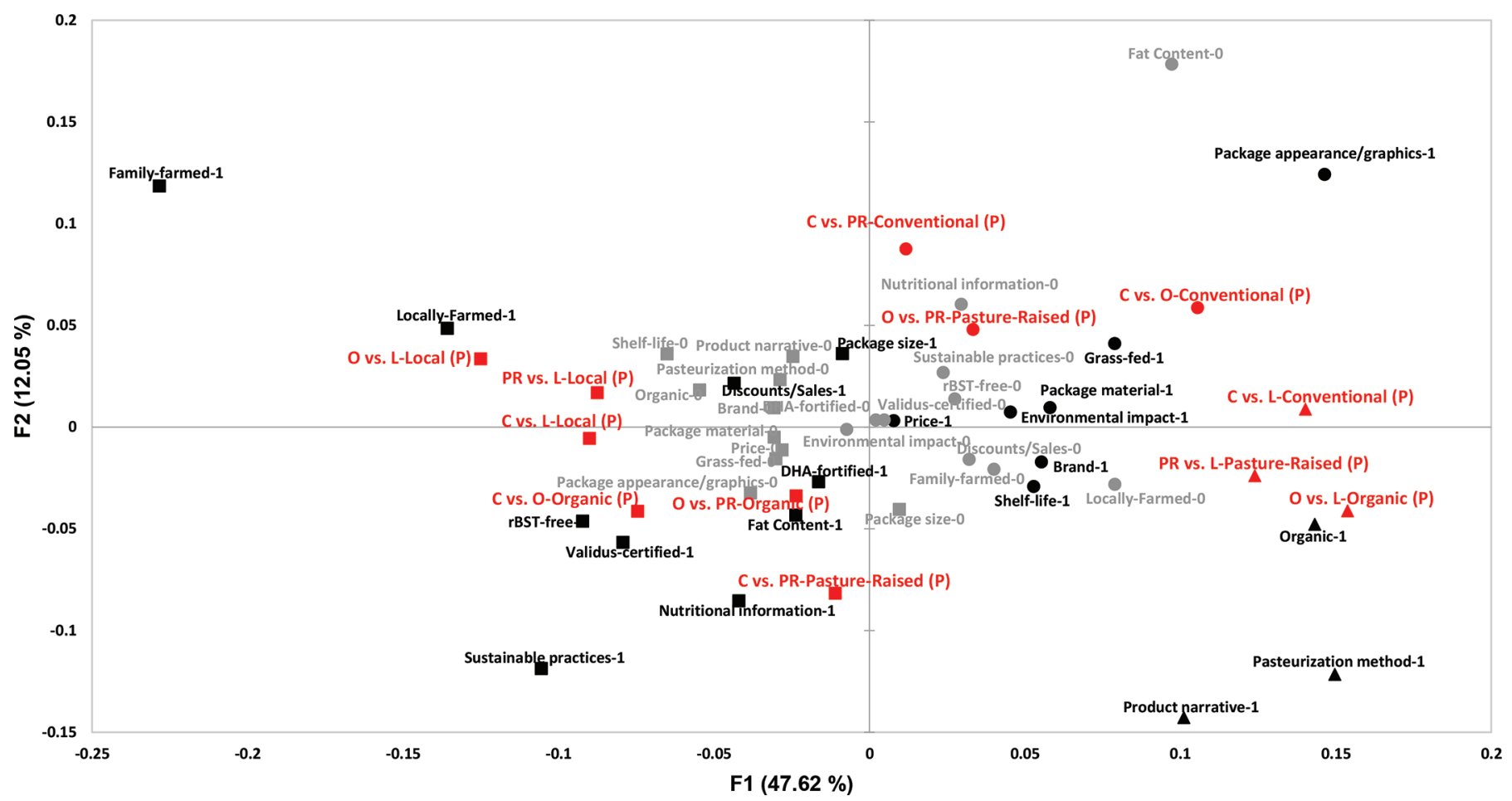

Figure 2. Multiple correspondence analysis of primed cohort $(\mathrm{n}=87)$ preference-test results and self-reported influences on fluid milk purchase. $\mathrm{C}=$ conventional; $\mathrm{O}=$ organic; $\mathrm{L}=$ locally farmed; $\mathrm{PR}=$ pasture raised; $\mathrm{P}=$ primed. Preference-test results are listed in the following order: pairing type, preference choice, priming treatment. $1=$ attribute was chosen in check-all-that-apply $($ CATA $)$ exercise, $0=$ attribute was not chosen in CATA exercise. $\mathbf{\square}=$ cluster 1 preferences and purchase influences; $\boldsymbol{\Lambda}=$ cluster 2 preferences and purchase influences, $\bullet=$ cluster 3 preferences and purchase influences. 
and Drake, 2018; Harwood and Drake, 2020). While the third cluster's proclivity toward pasture-raised and grass-fed appeared nonsensical, it should be noted that no clear preference pattern was expressed in favor of the pasture-raised milk type designation. Previous studies have shown "grass-fed" to be a universally attractive feature among different milk consumer groups (Harwood and Drake, 2018). Consequently, the present study suggests that the pasture-raised designation for fluid milk may lack a consumer-relevant identity relative to other "value-added" milk type designations and may be considered an inherent part of the milk production process, especially when decoupled from branding or package-related information and narrative. This conclusion is further evidenced by IAT exercise findings. Pasture-raised and organic milk type IAT comparisons showed that organic designation, a succinct and certifiable (USDA organic seal) quality indicator for fluid milk, was more often associated with a positive implicit response compared with pasture-raised. On the other hand, local designation, a product feature with inconsistent consumer understanding and beliefs and no formal definition, had no difference in implicit bias when compared with pasture-raised (Hand and Martinez, 2010).

\section{CONCLUSIONS}

Implicit association tests from fluid milk consumers revealed implicit biases against conventional fluid milk when compared with organic, locally farmed, or pasture-raised milk types. Similarly, an implicit bias in favor of organic over pasture-raised milk was reported. Evidence of bias-related influences on preference patterns were noted following primed and unprimed consumer evaluations of fluid milk types. For blinded evaluations, consumers differentiated fluid milk products primarily based upon flavor differences, which can be attributed to dissimilarities in packaging, processing, and feed flavors. Furthermore, there was minimal observed agreement between preference patterns and stated beliefs and purchase influences, indicating fluid milk consumers were largely unable to attribute such flavor distinctions with the associated milk type. On the other hand, preference patterns exhibited in primed evaluations showed product differentiation was primarily based upon agreement with belief systems, indicating that fluid milk consumers are, in general, likely to override sensory preferences to more closely align with stated beliefs. As such, this study serves as evidence that product narrative and consumer perceptions about farming practice are of chief concern for influencing milk type preference, and presumably, purchase. Specifically, findings from this study may be valuable to milk producers of conventional systems. Implicit association testing of fluid milk consumers clearly proves the existence of strong anticonventional biases. However, specific shortfalls in consumer perception of conventional milk such as animal welfare, sustainability, and lack of product narrative may be improved through alignment with locally farmed milk marketing and narratives, education initiatives, or general enhancement of product narrative through labeling. Furthermore, the present study may be a valuable resource for milk producers of all types (including those who use hybrid marketing approaches such as organic and pastureraised), to review or refine their marketing approach and identify product qualities that may be attractive or alienating to their target consumers.

\section{ACKNOWLEDGMENTS}

Funding was provided in part by The Dairy Alliance (Atlanta, GA) and the National Dairy Council (Rosemont, IL). The use of trade names does not imply endorsement or lack of endorsement of those not mentioned. The authors have not stated any conflicts of interest.

\section{REFERENCES}

Ares, G., A. Giménez, F. Bruzzone, L. Vidal, L. Antúnez, and A. Maiche. 2013. Consumer visual processing of food labels: Results from an eye-tracking study. J. Sens. Stud. 28:138-153. https://doi .org/10.1111/joss.12031.

Bernard, J. C., and Y. Liu. 2017. Are beliefs stronger than taste? A field experiment on organic and local apples. Food Qual. Prefer. 61:55-62. https://doi.org/10.1016/j.foodqual.2017.05.005.

Cardoso, C. S., M. J. Hötzel, D. M. Weary, J. A. Robbins, and M. A. von Keyserlingk. 2016. Imagining the ideal dairy farm. J. Dairy Sci. 99:1663-1671. https://doi.org/10.3168/jds.2015-9925.

Carpenter, T. P., R. Pogacar, C. Pullig, M. Kouril, S. Aguilar, J. LaBouff, N. Isenberg, and A. Chakroff. 2019. Survey-software implicit association tests: A methodological and empirical analysis. Behav. Res. Methods 51:2194-2208. https://doi.org/10.3758/ s13428-019-01293-3.

Croissant, A. E., S. P. Washburn, L. L. Dean, and M. A. Drake. 2007. Chemical properties and consumer perception of fluid milk from conventional and pasture-based production systems. J. Dairy Sci. 90:4942-4953. https://doi.org/10.3168/jds.2007-0456.

Friese, M., M. Wänke, and H. Plessner. 2006. Implicit consumer preferences and their influence on product choice. Psychol. Mark. 23:727-740. https://doi.org/10.1002/mar.20126.

Gandy, A. L., M. W. Schilling, P. C. Coggins, C. H. White, Y. Yoon, and V. V. Kamadia. 2008. The effect of pasteurization temperature on consumer acceptability, sensory characteristics, volatile compound composition, and shelf-life of fluid milk. J. Dairy Sci. 91:1769-1777. https://doi.org/10.3168/jds.2007-0833.

Getter, K. L., B. K. Behe, P. H. Howard, D. S. Conner, and L. M. Spaniolo. 2015. Increasing demand for pasture-based dairy: What attributes and images do consumers want? Pages 125-140 in ReThinking Organic Food and Farming in a Changing World. B. Freyer and J. Bingen, ed. Springer, Berlin, Germany.

Greenwald, A. G., B. A. Nosek, and M. R. Banaji. 2003. Understanding and using the implicit association test: I. An improved scoring algorithm. J. Pers. Soc. Psychol. 85:197-216. https://doi.org/10 .1037/0022-3514.85.2.197. 
Haas, R., A. Schnepps, A. Pichler, and O. Meixner. 2019. Cow milk versus plant-based milk substitutes: A comparison of product image and motivational structure of consumption. Sustainability 11:5046. https://doi.org/10.3390/su11185046.

Haas, R., J. Sterns, O. Meixner, D. I. Nyob, and V. Traar. 2013. Do US consumers perceive local and organic food differently? An analysis based on means-end chain analysis and word association. Int. J. Food Syst. Dyn. 4:214-226.

Hand, M. S., and S. W. Martinez. 2010. Just what does local mean? Choices. 25:1-4.

Harwood, W. S., and M. A. Drake. 2018. Identification and characterization of fluid milk consumer groups. J. Dairy Sci. 101:8860-8874. https://doi.org/10.3168/jds.2018-14855.

Harwood, W. S., and M. A. Drake. 2019. Understanding implicit and explicit consumer desires for protein bars, powders, and beverages. J. Sens. Stud. 34:e12493. https://doi.org/10.1111/joss.12493.

Harwood, W. S., and M. A. Drake. 2020. Validation of fluid milk consumer segments using qualitative multivariate analysis. J. Dairy Sci. In press.

Hill, H., and F. Lynchehaun. 2002. Organic milk: Attitudes and consumption patterns. Br. Food J. 104:526-542. https://doi.org/10 $.1108 / 00070700210434570$.

Kühl, S., B. Gassler, and A. Spiller. 2017. Labeling strategies to overcome the problem of niche markets for sustainable milk products: The example of pasture-raised milk. J. Dairy Sci. 100:5082-5096. https://doi.org/10.3168/jds.2016-11997.

Lee, A. P., D. M. Barbano, and M. A. Drake. 2017. The influence of ultra-pasteurization by indirect heating versus direct steam injection on skim and 2\% fat milks. J. Dairy Sci. 100:1688-1701. https: //doi.org/10.3168/jds.2016-11899.

Lim, J. 2011. Hedonic scaling: A review of methods and theory. Food Qual. Prefer. 22:733-747. https://doi.org/10.1016/j.foodqual.2011 .05 .008

McCarthy, K. S., M. N. Parker, A. Ameerally, S. L. Drake, and M. A. Drake. 2017. Drivers of choice for fluid milk versus plant-based alternatives: What are consumer perceptions of fluid milk? J. Dairy Sci. 100:6125-6138. https://doi.org/10.3168/jds.2016-12519.

Moskowitz, H. R., and J. L. Sidel. 1971. Magnitude and hedonic scales of food acceptability. J. Food Sci. 36:677-680. https://doi.org/10 $.1111 / j .1365-2621.1971 . t b 15160 . x$

Napolitano, F., C. Castellini, S. Naspetti, E. Piasentier, A. Girolami, and A. Braghieri. 2013. Consumer preference for chicken breast may be more affected by information on organic production than by product sensory properties. Poult. Sci. 92:820-826. https://doi .org/10.3382/ps.2012-02633.
Nosek, B. A., A. G. Greenwald, and M. R. Banaji. 2005. Understanding and using the Implicit Association Test: II. Method variables and construct validity. Pers. Soc. Psychol. Bull. 31:166-180. https: //doi.org/10.1177/0146167204271418.

Rizzo, P. V., W. S. Harwood, and M. A. Drake. 2020. Consumer desires and perceptions of lactose-free milk. J. Dairy Sci. 103:69506966. https://doi.org/10.3168/jds.2019-17940.

Rödiger, M., and U. Hamm. 2015. How are organic food prices affecting consumer behaviour? A review. Food Qual. Prefer. 43:10-20. https://doi.org/10.1016/j.foodqual.2015.02.002.

Rooth, D. O. 2010. Automatic associations and discrimination in hiring: Real world evidence. Labour Econ. 17:523-534. https://doi .org/10.1016/j.labeco.2009.04.005.

Scozzafava, G., F. Gerini, F. Boncinelli, C. Contini, E. Marone, and L. Casini. 2020. Organic milk preference: Is it a matter of information? Appetite 144:104477. https://doi.org/10.1016/j.appet.2019 .104477 .

Simon, M., and A. P. Hansen. 2001. Effect of various dairy packaging materials on the shelf life and flavor of ultrapasteurized milk. J. Dairy Sci. 84:784-791. https://doi.org/10.3168/jds.S0022 -0302(01)74534-1.

USDA-ERS (US Department of Agriculture- Economic Research Service). 2019. Data set: Fluid beverage milk sales quantities by product (annual). Accessed Aug. 25, 2020. https://www.ers.usda.gov/ webdocs/DataFiles/48685/fluidmilk.xlsx?v=3094.9

Vanhonacker, F., W. Verbeke, E. Van Poucke, and F. Tuyttens. 2007. Segmentation based on consumers' perceived importance and attitude toward farm animal welfare. Int. J. Sociol. Agric. Food 15:91-107.

Villeneuve, M. P., Y. Lebeuf, R. Gervais, G. F. Tremblay, J. C. Vuillemard, J. Fortin, and P. Y. Chouinard. 2013. Milk volatile organic compounds and fatty acid profile in cows fed timothy as hay, pasture, or silage. J. Dairy Sci. 96:7181-7194. https://doi.org/10 $.3168 /$ jds.2013-6785.

Wolf, C. A., G. T. Tonsor, and N. J. Olynk. 2011. Understanding US consumer demand for milk production attributes. J. Agric. Resour. Econ. 36:326-342.

\section{ORCIDS}

M. A. Drake @ https://orcid.org/0000-0002-4744-2493 